\title{
Nonampullary Duodenal Adenomas Rarely Recur after Complete Endoscopic Resection: A Swiss Experience Including a Literature Review
}

\author{
Piero V. Valli ${ }^{a} \quad J o a c h i m ~ C$. Mertens $^{a} \quad$ Amnon Sonnenberg $^{b} \quad$ Peter Bauerfeind $^{a}$ \\ aDivision of Gastroenterology and Hepatology, University Hospital Zurich, Zurich, Switzerland; bDivision of \\ Gastroenterology and Hepatology, Oregon Health and Science University, Portland, OR, USA
}

\section{Keywords}

Duodenal adenoma - Nonampullary duodenal adenoma - Duodenal polyposis - Endoscopic resection . Endoscopic treatment

\begin{abstract}
Introduction: Duodenal polyps and especially duodenal adenomas are a rare and mostly coincidental finding in patients undergoing upper gastrointestinal endoscopy. Due to their malignant potential, duodenal adenomas should be removed upon diagnosis. So far, the limited available data on the performance of endoscopic polypectomy show conflicting results with regard to adverse events and the adenoma recurrence rate. Patients and Methods: After summarizing the currently available data, we retrospectively analyzed all patients undergoing endoscopic resection of nonampullary duodenal adenomas (NAD) at our institution between 2006 and 2016. Results: A total of 78 patients underwent endoscopic polypectomy for NAD adenoma. End-of-treatment success with complete resection requiring a mean of 1.2 interventions was achieved in $91 \%(n=71)$. Procedural hemorrhage occurred in $12.8 \%$ ( $n=10)$, whereas delayed bleeding was noted in $9 \%(n=7)$. Duodenal perforation was registered and successfully treated in 2 cases (2.6\%). No adenoma recurrence was noted following primary complete adenoma resection after a mean follow-up time of 33 months. Acute post-polypectomy bleeding was statistically significantly as-
\end{abstract}

\section{KARGER}

(c) 2017 S. Karger AG, Basel

E-Mail karger@karger.com

www.karger.com/dig sociated with large polyp size $(p=0.003)$ and lack of endoscopic prophylaxis $(p=0.0008)$. Delayed post-polypectomy bleeding showed a trend in the occurrence of large polyps $(p=0.064)$, and was statistically significantly associated with familial cancer syndrome $(p=0.019)$ and advanced histopathology ( $p=0.013$ ). Conclusion: Our data suggest that endoscopic polypectomy of NAD is well feasible with high success rates. Procedural and delayed hemorrhage seems to be the primary issue rather than adenoma recurrence. We therefore advocate referral of patients with large NAD to experienced centers for endoscopic resection.

(c) 2017 S. Karger AG, Basel

\section{Introduction}

Duodenal adenomas are rare and mostly coincidental findings in patients presenting for routine upper gastrointestinal endoscopy. They can be divided into 3 subgroups: sporadically occurring duodenal adenomas or nonampullary adenomas of the duodenum (NAD), duodenal adenomas of the papilla of Vater, and duodenal adenomas associated with genetic polyposis syndromes such as familial adenomatous polyposis (FAP) or PeutzJeghers. Since duodenal adenomas bear a risk of malignant transformation comparable to colonic adenomas, removal is recommended upon diagnosis [1]. Due to the relatively low incidence of duodenal compared to colonic 


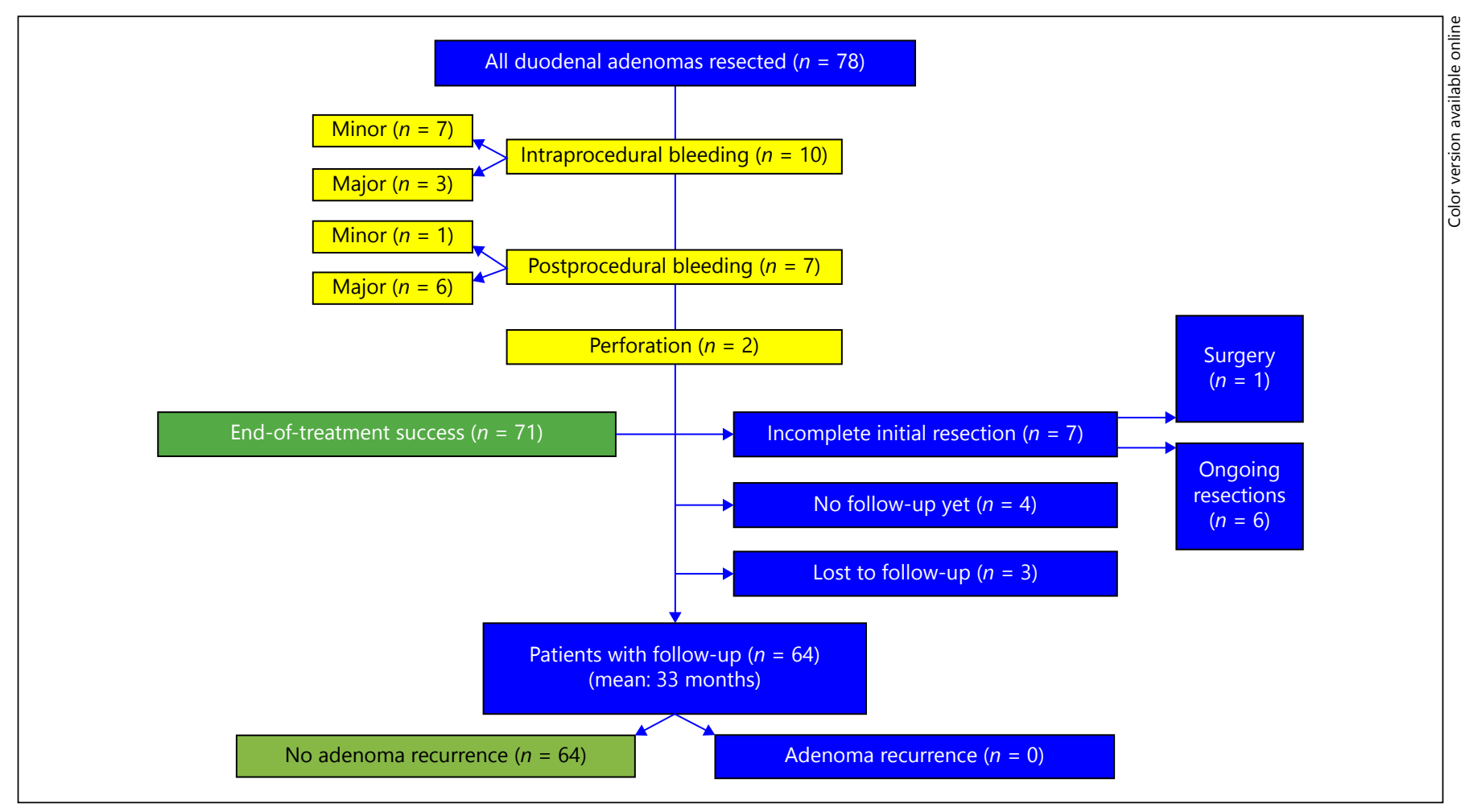

Fig. 1. Patient intervention and follow-up flowchart.

adenoma, large mortality studies are lacking. Until recently, the management of duodenal adenomas consisted of local or radical surgical resection, which carries a high morbidity and mortality risk as well a high recurrence rate. Recently, thanks to refined endoscopic techniques such as endoscopic mucosal resection (EMR), minimally invasive endoscopic management of such lesions has become the therapeutic approach of choice. Comparable to EMR in the right-sided colon, duodenal polypectomy is performed after lifting the lesion with submucosal liquid injection in order to counteract perforation of the thin duodenal wall. Different alternative resection techniques such as hybrid endoscopic submucosal dissection [2], cap-assisted suck and cut [3], underwater EMR [4], and band and slough [5] have been reported in small populations so far. In addition, remnants of adenomatous tissue after primary endoscopic resection may be removed by ablative interventions such as argon plasma coagulation (APC), radio frequency ablation (RFA), or electrocautery [6].

Data currently available on the endoscopic management of duodenal adenomas and its adverse events remain limited. Especially, the rates of adverse events and adenoma recurrence after polypectomy are very heteroge- neous among the few available publications. Recently, the American Society of Gastrointestinal Endoscopy has released their guidelines on the role of endoscopy in duodenal adenomas [7]. We here present a literature overview on the available literature and we analyzed the data of all patients $(n=78)$ who underwent EMR for duodenal adenoma at our institution between 2006 and 2016. The purpose of this study was to evaluate the efficacy and safety of EMR for NAD. We specifically evaluated the rate and risk factors of short-term (mainly bleeding and perforation) and longterm (bleeding) adverse events as well as the frequency of adenoma recurrence and the need for follow-up.

\section{Materials and Methods}

\section{Patients}

The primary endpoint of our study was to examine the outcome of EMR of duodenal polyps with regards to short-term (duodenal perforation, bleeding) and long-term (bleeding) adverse events as well as the frequency of adenoma recurrence. For this reason, we reviewed our endoscopic charts from 2006 to 2016. Seventy-eight patients (Fig. 1) met the inclusion criteria (patients with nonampullary duodenal polyp(s) undergoing endoscopic adenoma resection at our institution). Both sporadically occurring duodenal adenomas as well as adenomas in the context of 

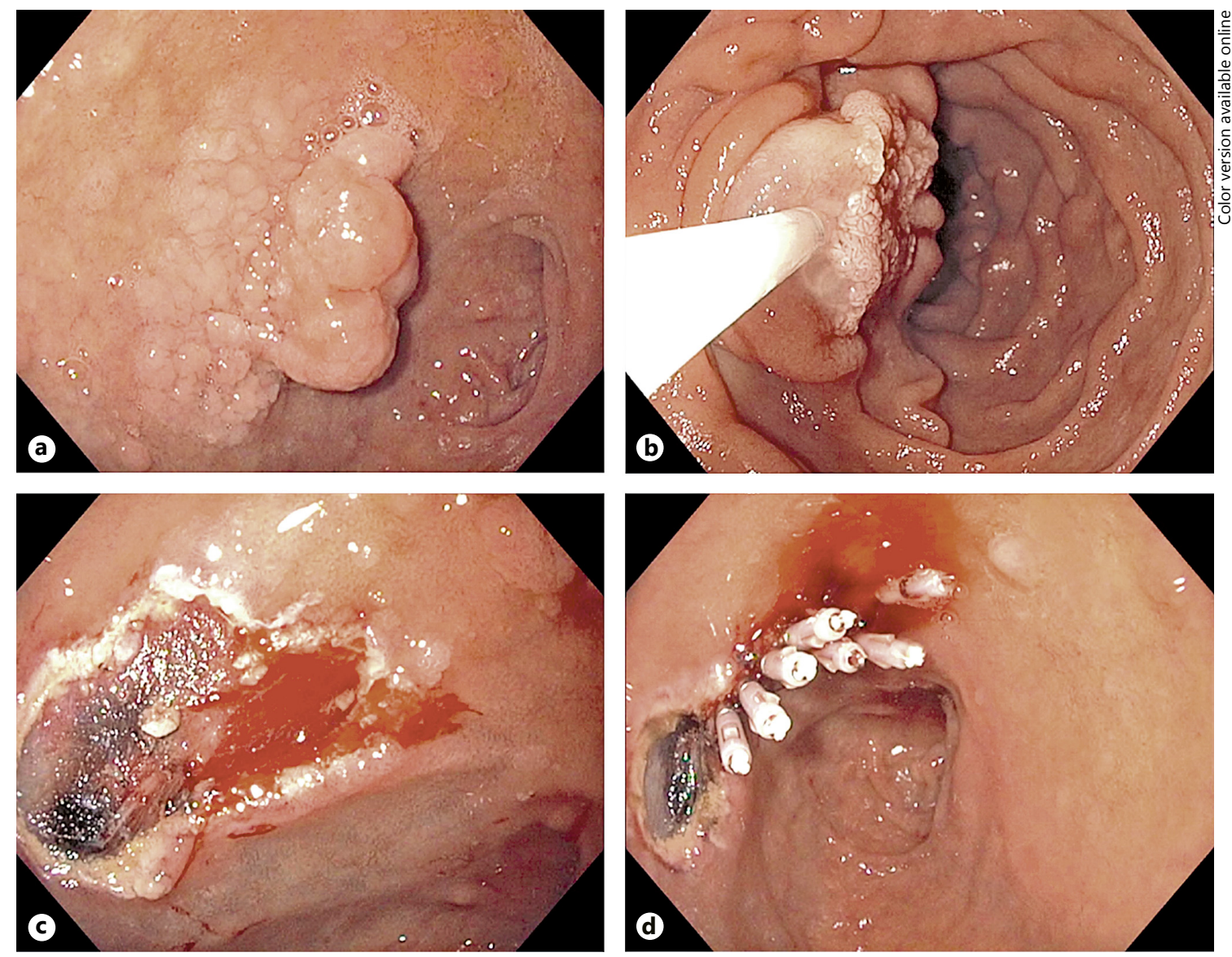

Fig. 2. a Endoscopic assessment of the location and size of the NAD. b Submucosal saline injection in order to create a cushion. c Snare polypectomy of the entire NAD. d Prophylactic adaption of polypectomy site using hemoclips.

FAP were included. All patients gave their written consent to the procedure and use of their medical records for research purposes. The study was approved by the local Ethics Committee (KEK ZH 2014-0465).

\section{Endoscopies and EMR}

All esophagogastroduodenoscopies were performed by 3 experienced endoscopists under non-anesthetist application of propofol using flexible standard Olympus ${ }^{\circledR}$ endoscopes. If possible, all anticoagulant or antithrombotic medications were discontinued 7 days prior to the intervention. After careful assessment of location, size, spreading, and involvement of the major papilla (side-viewing endoscope), adenoma resection was conducted using either a biopsy forceps or snare polypectomy with prior submucosal isotonic saline injection $(\mathrm{NaCl} 0.9 \%)$ without or with blue dye. If technically feasible, en bloc EMR was always aimed for. Snare resection was the preferred polypectomy technique (Fig. 2). In case of residual adenoma tissue on the resection margins, APC or RFA was conducted. Intravenous butylscopolamine was administered at the discretion of the endoscopist. Duodenal hemorrhage provoked by polypectomy were subdivided into " $\mathrm{mi}$ nor" (hemoglobin drop <2 g/L) and "major" (hemoglobin drop
$>2 \mathrm{~g} / \mathrm{L}$ and/or need for red blood cell transfusion) bleeding. We differentiated between immediate (during the polypectomy) or delayed bleeding (a second esophagogastroduodenoscopies was necessary in the follow-up during the first 2 weeks after polypectomy). If required, endoscopic hemostasis was achieved using standard hemoclips, over-the-scope clips $\left(\right.$ OTSC $^{\circledR}$, Ovesco, Tübingen), loop ligation (endo loop, Olympus ${ }^{\circledR}$ ) or argon plasma coagulation (APC, Erbe, Tübingen). In the absence of immediate hemorrhage, performed hemostatic interventions were considered prophylactic hemostasis.

Adenoma size was measured on the histopathological specimen. In case of piecemeal resection, the size of the largest pathological piece was recorded. After polypectomy, proton pump inhibitors were administered for at least 4 weeks. The majority of interventions were performed on an in-patient basis depending on the adenoma size, the difficulty of the procedure, and the comorbidities of the patient.

Follow-Up

The first follow-up endoscopy in case of complete resection was conducted 12 months after endoscopic polypectomy or at the discretion of the endoscopist. 
Table 1. Patient and lesion characteristics

\begin{tabular}{lccc}
\hline & $n$ & Range & Percentage \\
\hline Patients & 78 & & \\
Age, years, mean & 65.9 & $33-91$ & \\
Gender $\quad$ & & \\
$\quad$ Male & 47 & & 60.3 \\
$\quad$ Female & 31 & & 39.7 \\
Non-FAP & 70 & & 89.7 \\
FAP & 8 & & 10.3 \\
HNPCC & 0 & & 0.0 \\
Adenomas & 17.2 & $2-55$ & \\
Size, mm, mean & 62 & & 79.5 \\
Solitary & 16 & & 20.5 \\
Multifocal & & & 69.2 \\
Histopathology, $n$ & 54 & & 30.8 \\
$\quad$ Tubular & 24 & & \\
$\quad$ Tubulo-villous & 65.3 & 16.7 \\
Dysplasic grading & 13 & & \\
$\quad$ LGD & & \\
$\quad$ HGD & & & \\
\hline
\end{tabular}

Statistical Analysis

The statistical analysis was focused on factors that predicted the occurrence of acute or delayed bleeding and the presence of advanced histopathology. Advanced histopathology was defined as the presence of tubulovillous changes and low or high-grade dysplasia. In 3 separate logistic regressions, acute bleeding, delayed bleeding, and advanced histopathology served as 3 separate outcome variables. Demographic characteristics (age, gender), polyp characteristics (size, number, histopathology), resection technique (snare, biopsy, RFA, piecemeal vs. en bloc resection, prophylactic measures of hemostasis), and clinical history (prior bleeding, anticoagulation treatment) all served as predictor variables. The results of the multivariate analyses were assessed in terms of significant odds ratios or $p$ values associated with individual predictor variables.

\section{Results}

\section{Patients}

We here present a retrospective cohort of patients undergoing EMR of histologically confirmed adenomas of the duodenum performed at our institution, the largest tertiary referral center in Switzerland. A total of 78 patients (47 male [60\%] and 31 female [40\%]) with NAD underwent endoscopic removal (Table 1). The mean age of the population was 65.7 years. Eight out of the 78 adenomas (10\%) were associated with FAP, whereas 70 of the adenomas were sporadic (90\%).

\section{Adenoma Features}

The mean adenoma size was $16.8 \mathrm{~mm}$ (range $2-55 \mathrm{~mm}$ ). Sixty-two (79.5\%) adenomas were solitary, whereas 16 $(20.5 \%)$ were multifocal. Histopathological evaluation re- vealed 54 (69.2\%) tubular and $24(30.8 \%)$ tubulovillous adenomas. Sixty-five (83.3\%) were graded as low grade and the remaining $13(16.7 \%)$ as high grade dysplasia.

\section{Endoscopic Resection Features}

The majority $(n=72,92.3 \%)$ of the adenomas were hot snare resected after submucosal saline injection. The remaining $6(7.7 \%)$ polypectomies of small adenomas were performed using a standard biopsy forceps (Table 2). In $35.9 \%$ (28) en bloc resection was possible, whereas the remaining $64.1 \%$ (50) of the lesions were resected in a piecemeal technique. A mean of 1.2 (range 1-6) procedures were necessary to achieve a primary adenoma-free resection site in $91 \%$ (71) of patients. Six patients $(7.7 \%)$ remain under regular observation including repetitive endoscopic resection of remaining adenomatous tissue. In one patient (1.3\%), after an initial attempt to endoscopically resect a multitude of polyps, a secondary surgical resection had to be performed due to incomplete endoscopic removal. In 49 (62.8\%) of the cases, prophylactic hemostasis using hemoclips $(n=26$, $53.1 \%)$, APC $(n=8,16.3 \%)$, OTSC $^{\circledR}(n=6,12.2 \%)$, loop ligation $(n=1,2 \%)$ or a combination of these $(n=8$, $16.3 \%)$ was performed at the time of primary endoscopic resection.

\section{Adverse Events}

All of the 10 (12.8\%, 7 minor, 3 major) intraprocedural bleedings were stopped by endoscopic measures. In 7 cases (9\%), delayed bleeding (major 6, minor 1) was noticed after the primary intervention. Endoscopic hemostasis was then achieved in all cases. The mean time from the endoscopic adenoma resection to occurrence of delayed bleeding was $52 \mathrm{~h}$ (range 5-240 h). Of the patients with delayed bleeding, one patient underwent resection while still on a low-dose treatment with acetyl salicylic acid. A second patient had stopped the antiplatelet medication more than 7 days before the polypectomy. The remaining 5 patients did not take any anticoagulants. In 4 out of these 7 patients with delayed bleeding, prophylactic endoscopic hemostasis or hemostasis of a procedural bleeding had already been performed (APC and or clip application).

Two out of 78 (2.6\%) patients had a duodenal perforation that was successfully closed using an OTSC ${ }^{\circledR}$.

\section{Risk Factors for Adverse Events}

Acute post-polypectomy bleeding was statistically and significantly associated with large polyp size $(p=0.003)$ and lack of endoscopic prophylaxis $(p=0.0008)$. Delayed 
post-polypectomy bleeding showed a trend in large polyps $(p=0.064)$ and was statistically and significantly associated with familial cancer syndrome $(p=0.019)$ and advanced histopathology ( $p=0.013)$. Lastly, advanced histopathology was statistically significantly associated with large polyp size. In general, age and gender did not affect bleeding or polyp type.

\section{Follow-up}

Endoscopic long-term (mean 33 months, range 1-121) follow-up was available in 68 patients. During follow-up, there was no recurrence of duodenal adenoma in any of the patients who achieved complete endoscopic resection.

Three (3.8\%) patients were lost to follow-up after initial polypectomy. Follow-up was not yet due at the time of data analysis in 7 patients (9\%). Seven patients (9\%) did not achieve a primary complete adenoma removal and were therefore under regular surveillance for a longer time with repetitive endoscopic resections going on (Fig. 1).

\section{Review of the Literature}

A PubMed search was performed using the following Mesh-Terms: duodenal adenoma; nonampullary duodenal adenoma; duodenal polyposis; endoscopic resection; endoscopic treatment. After review of the abstracts, 17 publications were found to be clinically relevant and were then further analyzed (Table 3). A total of 519 patients were included in the 17 cohorts. The mean population size was 38 patients (range 12-106) with a mean followup of 26 months (range $0-100$ ).

One of the first publications was presented by Apel et al. [6] in 2005. Using a classic EMR technique after submucosal saline injections, a total of 21 polypectomies were performed. The authors did not report any shortterm adverse events, whereas delayed bleeding occurred in $9.5 \%$ of the cases. Adenoma recurrence rate upon follow-up was $25 \%$. Three years later, Lepilliez et al. [8] presented another retrospective analysis of endoscopically resected nonampullary duodenal adenomas. Among their 35 patients, the procedural bleeding rate was $14 \%$, while delayed bleeding occurred in $11.6 \%$. After a mean follow-up of 15 months, no adenoma recurrence was noted. Several other authors found an immediate bleeding rate in the same range $(6-18 \%)$ [3, 9-12]. In 2 studies, high rates of procedural hemorrhage (57.8 and $43 \%$, respectively) were observed $[13,14]$, whereas 3 authors did not observe any procedural bleeding complications $[2,5$, 15]. Duodenal perforation occurred in 8 cases among all
Table 2. Endoscopic treatment and follow-up

\begin{tabular}{|c|c|c|c|}
\hline Endoscopic treatment & $n$ & Range & Percentage \\
\hline Snare & 72 & & 92.3 \\
\hline Biopsy & 6 & & 7.7 \\
\hline En bloc & 28 & & 35.9 \\
\hline Piecemeal & 50 & & 64.1 \\
\hline Amount of EMR, mean & 1.2 & $1-6$ & \\
\hline Amount of EMR, median & 1 & & \\
\hline \multicolumn{4}{|l|}{ End of treatment success } \\
\hline Yes & 71 & & 91.0 \\
\hline Ongoing & 6 & & 7.7 \\
\hline Surgery & 1 & & 1.3 \\
\hline \multicolumn{4}{|l|}{$\begin{array}{l}\text { Prophylactic endoscopic } \\
\text { hemostasis }\end{array}$} \\
\hline No & 29 & & 37.2 \\
\hline Yes & 49 & & 62.8 \\
\hline APC & 9 & & 18.4 \\
\hline Hemoclip & 26 & & 53.1 \\
\hline OTSC & 6 & & 12.2 \\
\hline Loop & 1 & & 2.0 \\
\hline Combination & 8 & & 16.3 \\
\hline \multicolumn{4}{|l|}{ Adverse events } \\
\hline \multicolumn{4}{|l|}{ Procedural bleeding } \\
\hline No & 68 & & 87.2 \\
\hline Minor & 7 & & 9.0 \\
\hline Major & 3 & & 3.8 \\
\hline \multicolumn{4}{|l|}{ Endoscopic hemostasis } \\
\hline $\mathrm{APC}$ & 1 & & 10.0 \\
\hline Hemoclip & 5 & & 50.0 \\
\hline OTSC & 4 & & 40.0 \\
\hline \multicolumn{4}{|l|}{ Delayed bleeding } \\
\hline No & 71 & & 91.0 \\
\hline Minor & 1 & & 1.3 \\
\hline Major & 6 & & 7.7 \\
\hline Mean time to bleeding, $\mathrm{h}$ & 52 & & \\
\hline \multicolumn{4}{|l|}{ Endoscopic hemostasis } \\
\hline $\mathrm{APC}$ & 1 & & 14.3 \\
\hline Hemoclip & 3 & & 42.9 \\
\hline OTSC & 2 & & 28.6 \\
\hline Combination & 1 & & 14.3 \\
\hline \multicolumn{4}{|l|}{ Perforation } \\
\hline No & 76 & & 97.4 \\
\hline Yes & 2 & & 2.6 \\
\hline \multicolumn{4}{|l|}{ Treatment } \\
\hline OTSC & 2 & & 100.0 \\
\hline \multicolumn{4}{|l|}{ Follow-up } \\
\hline Incomplete initial resection & 7 & & 9.0 \\
\hline No follow-up yet & 4 & & 5.1 \\
\hline Lost to follow-up & 3 & & 3.8 \\
\hline Cases with a follow-up & 64 & & 82.1 \\
\hline Mean follow-up, months & 33 & $2-112$ & \\
\hline \multicolumn{4}{|l|}{ Adenoma recurrence } \\
\hline No & 64 & & 100.0 \\
\hline Yes & 0 & & 0.0 \\
\hline
\end{tabular}




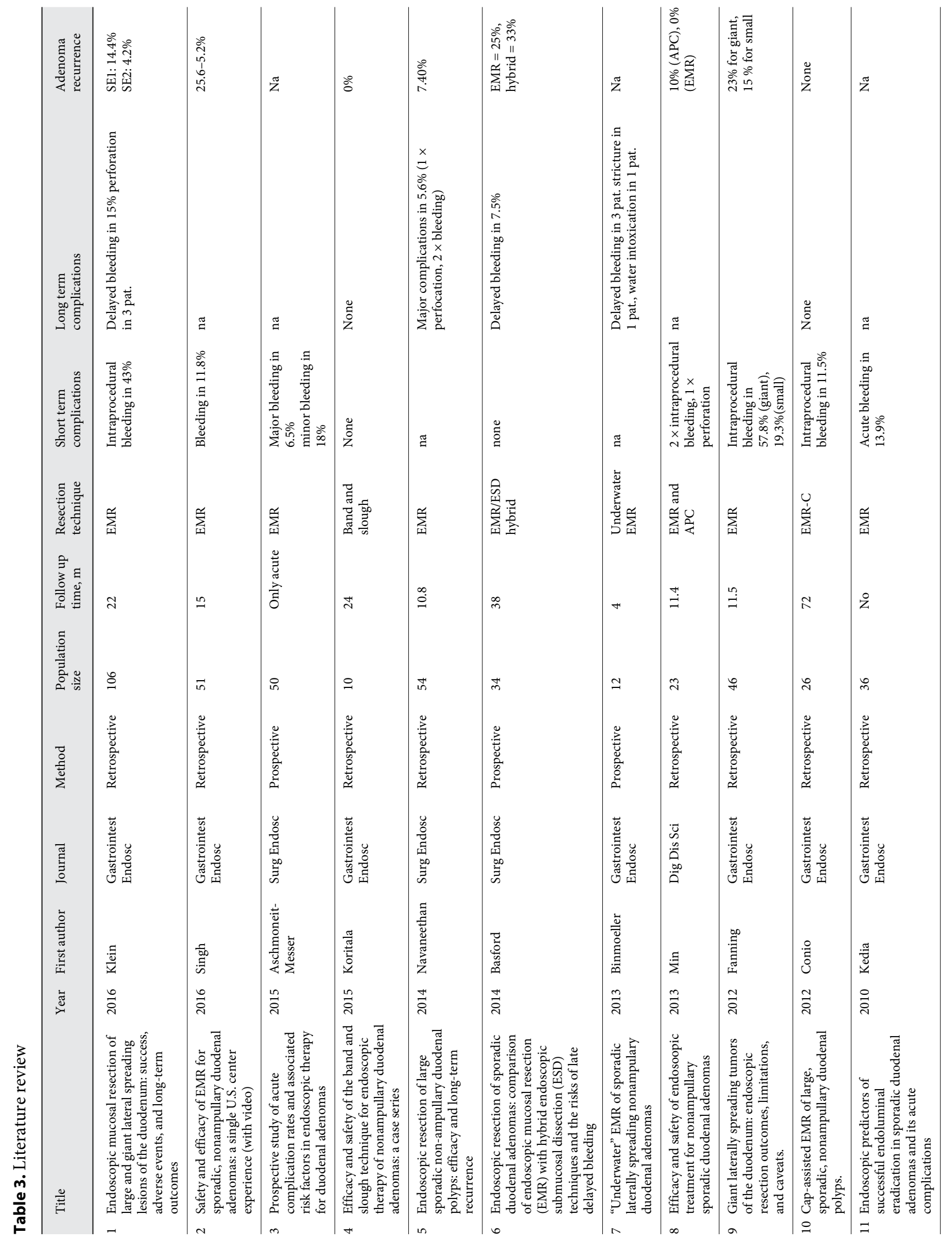




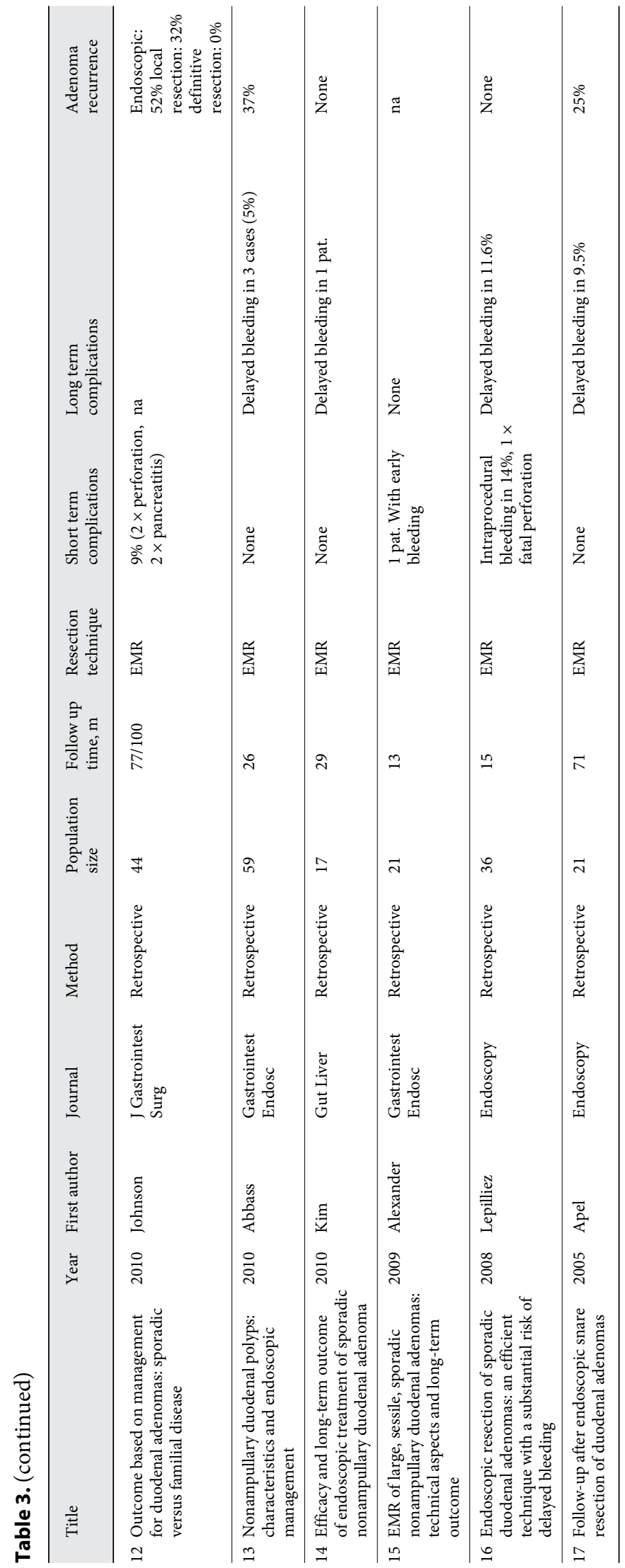

studies leading to an overall perforation of $1.5 \%[8,13$, $16-18]$. Delayed bleeding was noted in $7.5-15 \%$ in those studies that evaluated delayed adverse events. The incidence of adenoma recurrence showed substantial variation between 0 and $52 \%$ for a mean follow-up time of 23 months (range 0-100).

\section{Discussion}

Duodenal polyps are a relatively rare finding upon endoscopy in usually asymptomatic patients [19-22]. They consist of duodenal adenomas, gastric metaplasia, or submucosal lesions such as lipoma or GISTs. Even though little is known about the tumor biology of duodenal adenomas, they are believed to follow an adenoma-carcinoma sequence comparable to colonic adenomas [6]. Due to their malignant potential, duodenal adenomas occurring sporadically or in familial polyposis syndromes should be removed. Compared to tubular adenomas, tubulovillous lesions bear an increased risk to undergo malignant transformation [6]. In our cohort, approximately one third of the adenomas were tubulovillous. Historically, duodenal adenomas have been approached surgically, associated with substantial morbidity and mortality and with elevated recurrence rates most likely due to incomplete resection. Today, the approach of choice in limited disease should be endoscopic resection. Currently, there is only limited and mostly retrospective data available concerning the endoscopic removal of NAD. Despite the lack of large, prospective studies analyzing the efficacy and safety of endoscopic treatment of duodenal adenomas, the advantages such as accurate identification and visualization of complete resection, organ preservation, reduction of perioperative risks, recovery, and length of hospital stay are evident.

A significant portion of the patients undergoing polypectomy of NAD at our tertiary-care center were referrals from other gastroenterology centers. So far, we here present the largest cohort of NAD polypectomies in Switzerland.

In our cohort, we found a fairly high rate of immediate bleeding (14\%) compared to colonic polypectomy $[23,24]$ with classic bleeding rates below $5 \%$. This fact is most likely due to the abundant vascularization of the duodenal mucosa. The occurrence of acute post-polypectomy hemorrhage was statistically and significantly associated with large polyp size and the lack of endoscopic. Prophylactic hemostasis was performed in over 
$60 \%$ of our patients. Delayed bleeding occurred in almost $10 \%$ of the cases with a statistical trend in advanced adenoma histology and familial polyposis syndrome. Interestingly, no significant association was found between continued antithrombotic (low dose aspirin, $n=$ 4) medication and hemorrhage. In our experience, diffuse oozing was the predominant type of bleeding most likely due to the highly vascularized duodenal wall. Using standard through-the-scope clips seem less suitable for dealing with such bleeding. APC offers additional ablation of residual adenomatous tissues at the resection edges, yet bears the risk of transmural injury of the thin duodenal wall. The recently introduced OTSC ${ }^{\circledR}$ now offers an endoscopic tool for the compression of larger areas. Further studies need to assess which of the available tools for endoscopic hemostasis is the optimal one in case of duodenal polypectomy.

The frequency of perforation in our cohort was comparably low with $2.6 \%(2 / 76)$. Both duodenal perforations among our patients were successfully closed immediately after resection using an OTSC ${ }^{\circledR}$ and surgery was avoided. These findings underline an approximately 10 -fold increased perforation risk in the duodenum compared to the reported perforation rate of $0.07-0.13 \%$ in the ascending colon and the caecum [24].

In our cohort, we were able to confirm that the most relevant adverse event of endoscopic duodenal adenoma resection despite prophylactic measures is immediate and delayed bleeding from the polypectomy site. Given this relatively high frequency of adverse events compared to colonic polypectomy, we suggest that endoscopic resection of NAD should be performed in centers with expertise in the field.

Compared to a fairly high adenoma recurrence rate in other cohorts, our data show that once a duodenal adenoma is removed in toto, the risk of recurrence is very low to absent in the long-term follow-up (mean 3 years). We therefore believe that this discrepancy in recurrence rates is more likely due to the initial incomplete resection rather than adenoma recurrence. Yet, no consensus regarding surveillance intervals has been found among experts so far. To exhaustively address this question, larger cohorts are needed.

In conclusion, given the high rates of successful complete resection, our data support the opinion that sporadic and syndrome-associated NAD should be approached endoscopically. The most prevalent adverse event seems to be hemorrhage even in cases of prophylactic hemostasis. Furthermore, our data show that adenoma recurrence is less frequent than previously suggested in case of primary R0 resection.

\section{Disclosure Statement}

The authors hereby declare no conflicts of interest.

\section{References}

1 Seifert E, Schulte F, Stolte M: Adenoma and carcinoma of the duodenum and papilla of Vater: a clinicopathologic study. Am J Gastroenterol 1992;87:37-42.

2 Basford PJ, George R, Nixon E, et al: Endoscopic resection of sporadic duodenal adenomas: comparison of endoscopic mucosal resection (EMR) with hybrid endoscopic submucosal dissection (ESD) techniques and the risks of late delayed bleeding. Surg Endosc 2014;28:1594-1600.

3 Conio M, De Ceglie A, Filiberti R, et al: Capassisted EMR of large, sporadic, nonampullary duodenal polyps. Gastrointest Endos 2012;76:1160-1169.

4 Binmoeller KF, Shah JN, Bhat YM, et al: "Underwater" EMR of sporadic laterally spreading nonampullary duodenal adenomas (with video). Gastrointest Endosc 2013;78:496502.

5 Koritala T, Zolotarevsky E, Bartley AN, et al: Efficacy and safety of the band and slough technique for endoscopic therapy of nonampullary duodenal adenomas: a case series. Gastrointest Endosc 2015;81:985988.

6 Apel D, Jakobs R, Spiethoff A, et al: Follow-up after endoscopic snare resection of duodenal adenomas. Endoscopy 2005;37:444-448.

7 Committee ASoP, Chathadi KV, Khashab MA, et al: The role of endoscopy in ampullary and duodenal adenomas. Gastrointest Endosc 2015;82:773-781.

8 Lepilliez V, Chemaly M, Ponchon T, et al: Endoscopic resection of sporadic duodenal adenomas: an efficient technique with a substantial risk of delayed bleeding. Endoscopy 2008:40:806-810.

9 Kedia P, Brensinger C, Ginsberg G: Endoscopic predictors of successful endoluminal eradication in sporadic duodenal adenomas and its acute complications. Gastrointest Endosc 2010;72:1297-1301.

10 Aschmoneit-Messer I, Richl J, Pohl J, et al: Prospective study of acute complication rates and associated risk factors in endoscopic therapy for duodenal adenomas. Surg Endosc 2015;29:1823-1830.
11 Singh A, Siddiqui UD, Konda VJ, et al: Safety and efficacy of EMR for sporadic, nonampullary duodenal adenomas: a single US center experience (with video). Gastrointest Endosc 2016;84:700-708.

12 Alexander S, Bourke MJ, Williams SJ, et al: EMR of large, sessile, sporadic nonampullary duodenal adenomas: technical aspects and long-term outcome (with videos). Gastrointest Endosc 2009;69:66-73.

13 Klein A, Nayyar D, Bahin FF, et al: Endoscopic mucosal resection of large and giant lateral spreading lesions of the duodenum: success, adverse events, and long-term outcomes. Gastrointest Endosc 2016;84:688-696.

14 Fanning SB, Bourke MJ, Williams SJ, et al: Giant laterally spreading tumors of the duodenum: endoscopic resection outcomes, limitations, and caveats. Gastrointest Endosc 2012; 75:805-812.

15 Kim HK, Chung WC, Lee BI, et al: Efficacy and long-term outcome of endoscopic treatment of sporadic nonampullary duodenal adenoma. Gut Liver 2010;4:373-377. 
16 Navaneethan U, Lourdusamy D, Mehta D, et al: Endoscopic resection of large sporadic non-ampullary duodenal polyps: efficacy and long-term recurrence. Surg Endosc 2014;28: 2616-2622.

17 Min YW, Min BH, Kim ER, et al: Efficacy and safety of endoscopic treatment for nonampullary sporadic duodenal adenomas. Dig Dis Sci 2013;58:2926-2932.

18 Johnson MD, Mackey R, Brown N, et al: Outcome based on management for duodenal adenomas: sporadic versus familial disease. J Gas Surg 2010;14:229-235.
19 Ghazi A, Ferstenberg H, Shinya H: Endoscopic gastroduodenal polypectomy. Ann Surg 1984;200:175-180.

20 Hochter W, Weingart J, Seib HJ, et al: [Duodenal polyps. Incidence, histologic substrate and significance]. Dtsch Med Wochenschr 1984;109:1183-1186.

21 Jepsen JM, Persson M, Jakobsen NO, et al: Prospective study of prevalence and endoscopic and histopathologic characteristics of duodenal polyps in patients submitted to upper endoscopy. Scand J Gastroenterol 1994; 29:483-487.
22 Reddy RR, Schuman BM, Priest RJ: Duodenal polyps: diagnosis and management. J Clin Gastroenterol 1981;3:139-147.

23 Amato A, Radaelli F, Dinelli M, et al: Early and delayed complications of polypectomy in a community setting: the $\mathrm{SPoC}$ prospective multicentre trial. Dig Liver Dis 2016;48:4348.

24 Rutter MD, Nickerson C, Rees CJ, et al: Risk factors for adverse events related to polypectomy in the English bowel cancer screening programme. Endoscopy 2014;46:90-97. 